\title{
Relação entre gordura corporal, número de passos e capacidade cardiorrespiratória de mulheres na pós-menopausa
}

\author{
Relationship between body fat, number of steps and \\ cardiorespiratory fitness in postmenopausal women
}

Fabrício E. Rossi ${ }^{1}$, Camila Buonani ${ }^{1}$, Clara S. C. Rosa ${ }^{1}$, Tiego A. Diniz ${ }^{2}$, Diego G. D. Christofaro³ Ismael F. Freitas Junior ${ }^{4}$.

\begin{abstract}
RESUMO
Modelo do estudo: Estudo Transversal. Objetivos: Verificar a relação entre gordura corporal, número de passos e capacidade cardiorrespiratória em mulheres na pós-menopausa. Métodos: 57 mulheres acima dos 50 anos foram divididas em quatro grupos de acordo com a mediana da faixa etária $(<60,8 \geq$ 60,8 anos) e gordura corporal $(<22,8 \geq 33,2 \mathrm{Kg}$ ). Para estimativa da gordura corporal total, percentual de gordura e gordura de tronco, foi utilizado o DEXA. O teste de caminhada de seis minutos foi utilizado para analisar a capacidade cardiorrespiratória, e o número de passos por dia foi obtido por meio do acelerômetro tri-axial. Resultados: As mulheres jovens acumularam mais passos por dia $(<60,8=7727,5 \times \geq 60,8=6173,2$; $p<0,014)$ e as mulheres mais jovens com menor gordura corporal foram as que acumularam mais passos/dia (menor gordura $=8405$ passos $x$ alta gordura= 6118 passos; $p<0,031$ ), não diferindo nas mulheres com maior idade e maior gordura. Quanto à capacidade cardiorrespiratória, não houve diferença significativa entre os grupos. Todas as variáveis de gordura corporal apresentaram relação baixa e negativa com o número de passos no grupo mais jovem e com a capacidade cardiorrespiratória no grupo com idade mais avançada. Conclusão: A gordura corporal apresenta relação baixa e negativa com o número de passos e a capacidade cardiorrespiratória de mulheres na pós-menopausa, além disso, a idade parece influenciar nessa relação.
\end{abstract}

Palavras chave: Obesidade. Acelerometria. Atividade Física. Pós-Menopausa.

\section{Introduçåo}

O processo de envelhecimento é fator contribuinte para alterações fisiológicas e morfológicas importantes, como aumento de peso e da adiposidade corporal, principalmente a central ${ }^{1}$, além de redução da prática de atividade física e capacidade cardiorrespiratória ${ }^{2}$, que no caso das mulheres podem acentuar-se com a chegada da menopausa.

Sabe-se, que o excesso de gordura corporal, principalmente no tronco é fator independente para o desenvolvimento de distúrbios metabólicos, como re-
1. Pós-Graduando do Instituto de Biociências, Universidade Estadual Paulista (UNESP), Rio Claro, São Paulo, Brasil

2. Graduando do Curso de Educação Física da UNESP, Presidente Prudente, São Paulo, Brasil

3. Docente, Departamento de Educação Física, UNESP, Presidente Prudente, São Paulo, Brasil

4. Livre-Docente e Professor Associado, Departamento de Educação Física, UNESP, Presidente Prudente, São Paulo, Brasil
CORRESPONDÊNCIA

Fabrício E. Rossi,

Departamento de Educação Física, UNESP, Rua Roberto Simonsen, no 305 - Centro Educacional CEP 19060-900: Presidente Prudente, SP, Brasil,

Artigo recebido em 12/11/2013 Aprovado para publicação em 24/02/2014 
sistência à insulina, diabetes mellitus, dislipidemias e síndrome metabólica, contribuindo para o desenvolvimento de doenças cardiovasculares ${ }^{3,4}$; e elevação da taxa de mortalidade decorrente dessas doenças. ${ }^{5}$

Percentuais elevados de gordura corporal somado ao sedentarismo podem desencadear além de todas essas alterações metabólicas, diminuições significativas na mobilidade e velocidade de caminha$\mathrm{da}^{6}$, que são atividades de grande importância para a independência e boa qualidade de vida em idosos, tornando-se clara a importância de se prevenir o excesso de gordura corporal nessa fase da vida.

Estudos têm demonstrado que o aumento da prática habitual de atividade física, é eficaz para atenuar os efeitos negativos do envelhecimento e pósmenopausa $^{7}$ e que intervenções em longo prazo podem reduzir em até $9 \%$ o risco de obesidade ${ }^{8}$, evitando não só o acúmulo de gordura total, mas também na região do tronco ${ }^{9}$, no entanto, a situação inversa, ou seja, a relação da gordura corporal sobre o nível de atividade física e capacidade cardiorrespiratória em mulheres na pós-menopausa ainda não está totalmente esclarecida na literatura científica.

Sendo assim, o objetivo do presente estudo foi verificar a relação da gordura corporal sobre o número de passos e capacidade cardiorrespiratória em mulheres na pós- menopausa, além de verificar a influência da idade sobre esse desfecho.

\section{Material e métodos}

\section{Amostra}

Do ponto de vista ético, o presente estudo foi aprovado pelo Comitê de Ética e Pesquisa da Universidade Estadual Paulista, protocolo (64/2011). Todos os indivíduos incluídos neste estudo assinaram termo de consentimento livre e esclarecido. Estudo de delineamento transversal foi realizado nos anos de 2011 e 2012, na cidade de Presidente Prudente - SP. As voluntárias que concordaram em participar deste estudo fizeram parte de um programa de exercícios promovido pelo Departamento de Educação Física da Universidade Estadual Paulista (UNESP), Campus Presidente Prudente, SP, Brasil. A divulgação deste projeto foi realizada por meio da mídia local (jornais, televisão e rádio).

Um total de 197 mulheres foram avaliadas, mas somente 121 atenderam aos critérios de inclusão estabelecidos no estudo. Após a avaliação do número de passos, capacidade cardiorrespiratória e composição corporal, a amostra final foi selecionada por conveniência, devido nem todas as voluntárias terem realizado as avaliações pré determinadas e pela especificidade dessa população foi composta por 57 mulheres, na pós-menopausa, com idade superior a 50 anos (média $61,0 \pm 7,0$ anos), obesas ( $>35 \%$ gordura corporal), as quais foram divididas em quatro grupos de acordo com a mediana da faixa etária: mais jovens ( $<60,8$ anos) e com idade mais avançada ( $\geq 60,8$ anos) e posteriormente classificadas com menor gordura corporal, as voluntárias que estivessem a baixo da mediana da gordura corporal $(<22,8 \mathrm{Kg})$, e maior gordura corporal, as que se estivessem a cima da mediana ( $\geq 33,2 \mathrm{Kg}$ ). Para serem incluídas no projeto as participantes deveriam atender os seguintes critérios: i) estar na pós-menopausa (não ter ciclo menstrual por um ano ou mais $)^{10}$; ii) ter idade entre 50 e 79 anos na data da avaliação; iii) ser obesa; iv) não ter participado de programas de treinamento físico há pelo menos seis meses; v) não fazer terapia de reposição hormonal; vi) assinar o Termo de Consentimento Livre e Esclarecido, formalizando sua participação no estudo.

\section{Coleta de Dados}

\section{Antropometria e Composição Corporal}

A estatura foi mensurada por meio de um estadiômetro fixo (Sanny), com precisão de $0,1 \mathrm{~cm}$ e extensão máxima de 2,2 m. A medida de massa corporal foi realizada utilizando uma balança digital (Filizola), com precisão de $0,1 \mathrm{~kg}$ e capacidade máxima de $180 \mathrm{~kg}$. Todas as medidas foram realizadas seguindo padronização descrita por Freitas Jr. et al ${ }^{11}$.

A composição corporal foi estimada por meio da técnica de Dual-Energy X-ray Absorptiometry (DEXA) scanner version 4.7 (General Electric Healthcare, Lunar DPX-NT; England, a qual foi utilizada para mensurar a gordura corporal total, expressa em kg e valores percentuais, e gordura de tronco, expressa em $\mathrm{kg}$.

\section{Avaliação da Capacidade Cardiorrespiratória}

O teste de caminhada de seis minutos (TC6') foi aplicado sempre pelos mesmos examinadores em um corredor de 30 metros, com marcações a cada 5 metros, para exata determinação da distância percorrida. ${ }^{12}$ As voluntárias foram monitorizadas pelo cardiofrequencímetro Polar S810i e o índice de percepção da intensidade de esforço ${ }^{13}$ foi obtido no final do 
teste. Todas as voluntárias foram orientadas a andar em sua velocidade máxima durante os seis minutos do teste; se necessário elas poderiam parar para descansar e em seguida retomar o teste. Os examinadores utilizaram frases de incentivo para que as voluntárias mantivessem o mesmo ritmo de caminhada até o final do teste, quando foi medida a distância percorrida.

\section{Avaliação do Número de Passos Diário}

Para análise do número de passos, foi utilizado um sensor de movimento tipo acelerômetro tri-axial marca Actigraph, modelo GT3X (Actigraph LLC, Pensacola, FL). As recomendações de uso do acelerômetro foram realizadas por instrutores previamente treinados e familiarizados com o aparelho, as quais compunham: i) utilizar o acelerômetro por sete dias seguidos, todas as horas do dia em que permanecer em vigília; ii) retirar apenas para higiene pessoal, atividades aquáticas e para dormir; iii) posicionar preso à cintura do lado direito. Para análise dos dados foi utilizado o software específico, ActiLife 5 - Data Analysis Software by Actigraph. Foram incluídos no banco de dados apenas dias com mais de 10 horas de uso, os quais foram considerados, completos. Ao final, cada participante do estudo deveria ter no mínimo cinco dias completos de monitoramento, e o número de passos por dia foi calculado a partir da divisão do número total de passos semanais pelo número de dias utilizados.

\section{Análise dos dados}

Para o tratamento estatístico foi utilizado o teste de Kolmogorov-Smirnov para testar a normalidade do conjunto de dados e, com base nos parâmetros fornecidos, foi realizada estatística paramétrica e os dados foram apresentados em valores de média e desvio-padrão. A diferença entre o grupo com alta e baixa gordura corporal foi testado pelo teste $t$ para amostras independentes e para verificar a relação entre as variáveis de composição corporal e capacidades motoras, o teste de correlação de Pearson foi utilizado. Todas as análises foram realizadas por meio do software estatístico SPSS (versão 17.0). O nível de significância adotado foi de $5 \%$.

\section{Resultados}

A Tabela 1 apresenta as características gerais da amostra em valores de média e desvio padrão, bem como a comparação entre os grupos de acordo com a faixa etária, grupo abaixo da mediana $(<60,8$ anos; $n=27$ ) e grupo acima da mediana ( $\geq 60,8$ anos; $n=30$ ).

Quando comparados de acordo com a faixa etária, pode-se observar que os grupos apresentaram diferença estatisticamente significante em relação à idade e no número de passos, pois as mulheres com idade inferior a 60,8 anos caminharam em média 1554 passos a mais em relação ao grupo com idade mais avançada.

Tabela 1. Características gerais da amostra e comparação de acordo com a faixa etária.

\begin{tabular}{|c|c|c|c|c|c|c|c|c|c|c|}
\hline \multirow{2}{*}{$\begin{array}{l}\text { Variáveis } \\
\text { Idade (anos) }\end{array}$} & \multicolumn{3}{|c|}{$\begin{array}{c}\text { Amostra Total } \\
(\mathrm{n}=57)\end{array}$} & \multicolumn{3}{|c|}{$\begin{array}{c}<60,8 \text { anos } \\
(\mathbf{n}=27)\end{array}$} & \multicolumn{3}{|c|}{$\begin{array}{c}\geq \underset{60,8 \text { anos }}{(n=30)} \\
\end{array}$} & \multirow{2}{*}{$\begin{array}{c}\mathbf{p} \\
<0,001\end{array}$} \\
\hline & 61,0 & \pm & 7,0 & 55,4 & \pm & 3,2 & 66,1 & \pm & 5,3 & \\
\hline Peso (kg) & 71,4 & \pm & 14,6 & 74,8 & \pm & 16,2 & 68,3 & \pm & 12,6 & 0,125 \\
\hline Estatura $(\mathrm{cm})$ & 155,8 & \pm & 7,3 & 156,9 & \pm & 7,0 & 154,8 & \pm & 7,6 & 0,095 \\
\hline GC (kg) & 32,0 & \pm & 9,6 & 33,7 & \pm & 10,7 & 30,6 & \pm & 8,5 & 0,354 \\
\hline GC (\%) & 44,8 & \pm & 5,7 & 44,7 & \pm & 5,5 & 45,0 & \pm & 5,8 & 0,844 \\
\hline GT (kg) & 16,1 & \pm & 6,2 & 17,8 & \pm & 5,6 & 15,8 & \pm & 4,5 & 0,151 \\
\hline Passos por dia & 6909,5 & & 2414,3 & 7727,5 & \pm & 2559,1 & 6173,2 & \pm 2 & 049,2 & 0,014 \\
\hline TC6' (m) & 519,4 & \pm & 70,2 & 534,2 & \pm & 76,6 & 506,1 & \pm & 62,2 & 0,133 \\
\hline
\end{tabular}

$\mathrm{GC}(\mathrm{kg})=$ gordura corporal total em quilogramas; $\mathrm{GC}(\%)=$ percentual de gordura corporal; $\mathrm{GT}$ = gordura de tronco em quilogramas; TC6'= teste de caminhada de seis minutos. 
Os Gráficos 1 e 2 mostram as análises do número de passos realizados por dia e na capacidade cardiorrespiratória de acordo com a gordura corporal total. Pode-se observar no Gráfico 1, que as mulheres com idade inferior a 60,8 anos e que apresentaram menor gordura corporal foram as que acumularam maior número de passos/dia (menor gordura $=$ 8405 passos $\mathrm{x}$ maior gordura $=6118$ passos), não diferindo nos demais grupos. Em relação à capacidade cardiorrespiratória (Gráfico 2), não houve diferença estatisticamente significante entre os grupos.

Na Tabela 2, podem ser observados os valores de correlação entre o número de passos realizados por dia, capacidade cardiorrespiratória e gordura corporal total, percentual de gordura e gordura de tronco. Como foi verificada a influência da idade sobre o número de passos, testou-se a correlação em cada grupo separadamente.

Em relação ao número de passos houve correlação baixa e negativa somente na faixa etária abaixo de 60,8 anos com todas as variáveis de gordura corporal e associação baixa e positiva com capacidade cardiorrespiratória no grupo com idade mais avançada. Em relação à capacidade cardiorrespiratória, também houve correlação baixa e negativa com todas as variáveis de composição corporal, no entanto apenas no grupo com idade $\geq 60,8$ anos.

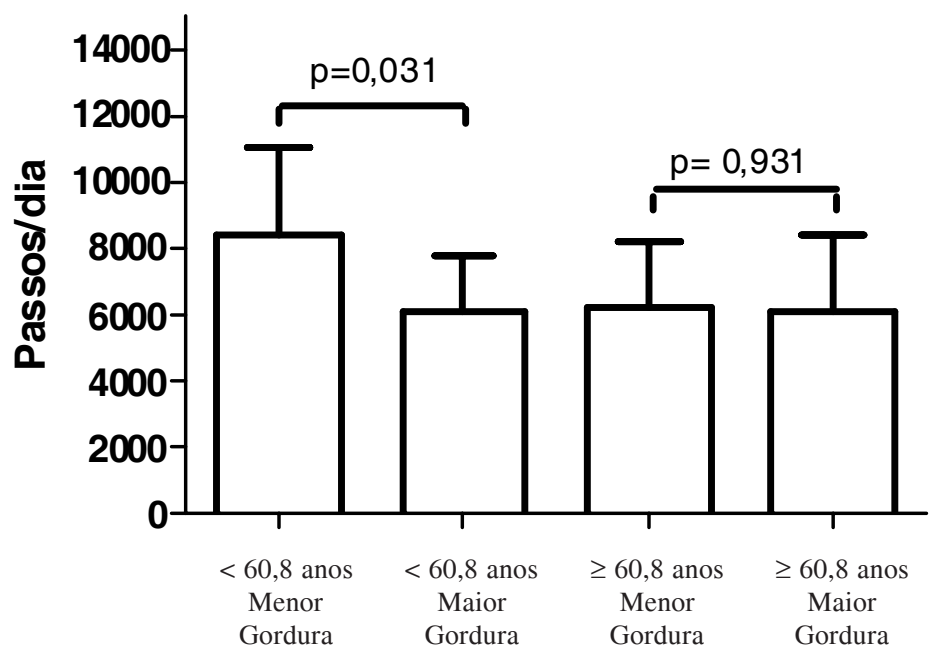

Gráfico 1. Análise do número de passos realizados por dia de acordo com a gordura corporal total em mulheres na pós-menopausa.

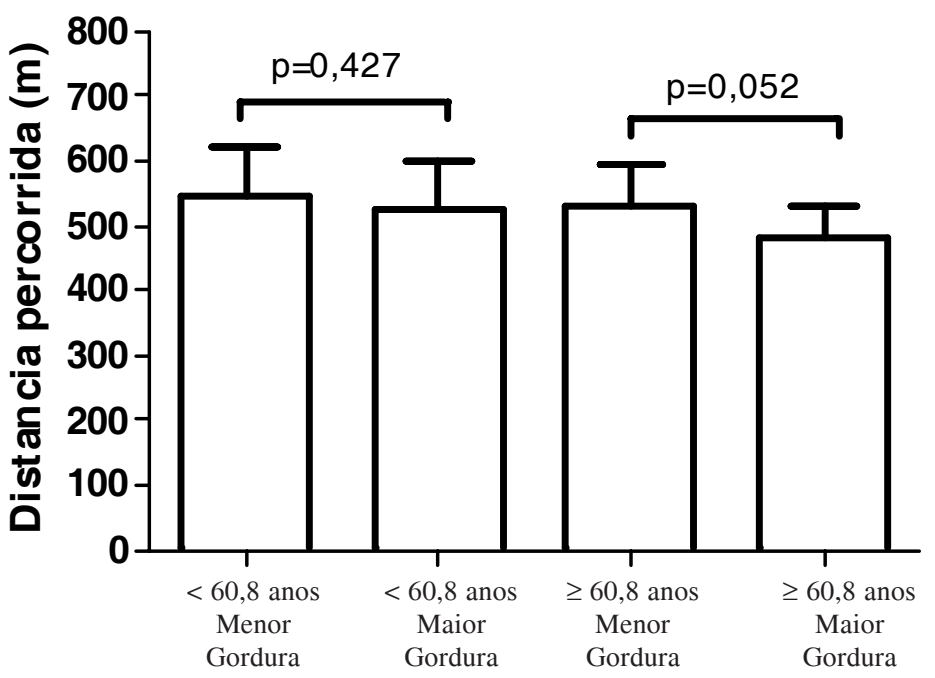

Gráfico 2. Análise da capacidade cardiorrespiratória de acordo com a gordura corporal total em mulheres na pós-menopausa.

Tabela 2: Correlação entre número de passos, capacidade cardiorrespiratória, gordura corporal total, percentual de gordura e gordura de tronco.

\begin{tabular}{|c|c|c|c|c|}
\hline \multirow[b]{2}{*}{ Variáveis } & \multicolumn{2}{|c|}{ Passos/dia } & \multicolumn{2}{|c|}{ TC6' } \\
\hline & $<61$ anos & $\geq 61 \mathrm{anos}$ & $<61$ anos & $\geq 61 \mathrm{anos}$ \\
\hline GC (kg) & $-0,495 * *$ & $-0,023$ & $-0,312$ & $-0,402 *$ \\
\hline GC (\%) & $-0,503^{*}$ & $-0,030$ & $-0,222$ & $-0,394^{*}$ \\
\hline GT (kg) & $-0,493^{*}$ & 0,028 & $-0,222$ & $-0,512 *$ \\
\hline TC6' (m) & 0,149 & $0,440 *$ & 1,000 & 1,000 \\
\hline
\end{tabular}

${ }^{*} p<0,05 ;{ }^{* *} p<0,001 ;$ passos/dia= passos realizados por dia; TC6'= teste de caminhada de seis minutos; GC $(\mathrm{kg})=$ gordura corporal total em quilogramas; $\mathrm{GC}(\%)=$ percentual de gordura corporal; $\mathrm{GT}=$ gordura de tronco em quilogramas. 


\section{Discussão}

Estudo de delineamento transversal que teve como objetivo verificar a relação da gordura corporal com o número de passos e capacidade cardiorrespiratória em mulheres na pós-menopausa, além de verificar a influência da idade sobre esse desfecho. Sendo a faixa etária um dos possíveis fatores que podem contribuir para a interferência nas variáveis analisadas no presente estudo, tornando-se assim um fator de confusão ${ }^{14}$, a mesma foi controlada nesse trabalho, minimizando a influência deste fator.

No presente estudo, observou-se que as mulheres mais jovens $(<60,8$ anos) apresentaram um acúmulo no número de passos significativamente superior às mulheres com idade mais avançada ( $\geq 60,8$ anos). Pode-se ressaltar como um dos achados mais importantes deste trabalho é de que as participantes mais jovens que apresentaram menor quantidade de gordura corporal foram as que acumularam maior número de passos (Gráfico 1), sugerindo que nessa faixa de idade, a quantidade de gordura pode interferir na prática habitual de atividade física. Corroborando com nossos achados, Buonani et al. ${ }^{15}$, verificaram que mulheres na menopausa que apresentaram valores superiores dos minutos em atividades moderada e vigorosa, e dos counts (unidade de medida arbitrária gerada pelo acelerômetro) de atividade física total quando avaliadas também por meio de acelerômetro tri-axial obtiveram níveis inferiores de gordura corporal, mostrando que o aumento na prática de atividade física durante essa fase da vida pode ser de grande importância na redução da gordura corporal, evitando assim um quadro de obesidade e doenças crônico-degenerativas como diabetes tipo 2 , hipertensão e síndrome metabólica. ${ }^{16}$

Em relação à capacidade cardiorrespiratória (Gráfico 2), novamente o grupo com menor gordura corporal percorreu maior distância no TC6', embora essa diferença não tenha sido estatisticamente significante. Corroborando com nossos achados, Freitas Junior et al. ${ }^{11}$ em estudo realizado também com mulheres na pós-menopausa, verificaram que as participantes que percorreram uma maior distância no TC6' apresentaram valores diminuídos de IMC, circunferência de cintura e prega tricipital, quando comparado ao grupo de menor distância.

Woo et al. ${ }^{17}$ verificaram a influência da gordura corporal, no entanto, analisando a velocidade de caminhada de seis metros ao invés da distância per- corrida como no presente estudo, em homens e mulheres com idade de 65 anos ou mais, e observaram que o grupo de pessoas com obesidade tinha velocidade média menor quando comparado ao grupo não obeso, e apresentou tempo 13\% maior para concluir o teste. Angleman et al. ${ }^{18}$, em estudo conduzido com 1030 mulheres e 888 homens com idade entre 55-74 anos, também observaram velocidade de caminhada mais lenta e prevalente em mulheres obesas em comparação com as não obesas $(31,7 \%$ e $14,6 \%$, respectivamente).

Uma das possíveis explicações para que isso possa acontecer é pelo fato de que a atividade aeróbica, caracterizada principalmente pela caminhada, pode contribuir para o aumento da capacidade oxidativa, das atividades das enzimas aeróbias, estoques de glicogênio intramuscular, densidades mitocondriais e capilares nos músculos, gasto energético de repouso, consumo máximo de oxigênio, melhorando consequentemente a capacidade cardiorrespiratória ${ }^{19}$ e resultando na diminuição de gordura corporal total e de tronco. ${ }^{9}$

Adicionalmente, foi observada nesse estudo, relação inversa de todas as variáveis de composição corporal analisadas com o número de passos no grupo mais jovem e com a capacidade cardiorrespiratória no grupo com idade mais avançada (Tabela 2), sugerindo que em mulheres com idade superior a 60 anos, a capacidade cardiorrespiratória esta mais relacionada à redução da gordura corporal total e central, do que o número de passos realizados por dia.

Resultados próximos foram observados por Thomson et al. ${ }^{20} \mathrm{em}$ acompanhamento realizado com 40 homens e 229 mulheres em que verificaram relação inversa entre o número de passos/dia e percentual de gordura corporal. Graff et al. ${ }^{21}$ em um estudo com o uso de pedômetro, no qual avaliaram mulheres de 15 a 35 anos, saudáveis, também verificaram associação negativa do nível de atividade física, com IMC, circunferência de cintura, percentual de gordura avaliado por dobras, além de observar também melhora na sensibilidade à insulina e índice HOMA, indicando que maior nível de atividade física pode influenciar tanto a melhora na composição corporal como também no perfil metabólico em diferentes populações.

Apesar da importância dos achados do presente estudo é necessário mencionar suas limitações. Primeiro, o tamanho amostral reduzido que indica a necessidade de cautela na extrapolação dos resultados. Segundo, o desenho transversal do estudo que impossibilita qualquer consideração sobre a relação de causa 
e efeito, sugerindo assim, estudos longitudinais de intervenção para verificar o efeito do excesso de gordura corporal sobre o número de passos e capacidade cardiorrespiratória. Terceiro, a intensidade das atividades não foram consideradas, fator esse que limita maior conhecimento sobre o esforço demandado pelas participantes do projeto.

Entretanto, devem-se ressaltar que a mensuração do número de passos foi realizada por meio de acelerômetro tri-axial, evitando, assim, o erro da medição de auto-relato ${ }^{22}$, além disso, com avaliação realizada pelo acelerômetro mais avançado (tri-axial quando comparado ao uni-axial) fatores como inclinação são considerados. Adicionalmente, a utilização do DEXA para estimar a composição corporal é outro aspecto diferencial, visto que é um método de alta precisão e fidedigno para a população idosa e que também proporciona resultados de composição corporal por meio de segmentos corporais ${ }^{23}$, possibilitando dessa forma a análise de gordura tronco de forma isolada.

\section{Conclusão}

Houve correlação baixa e negativa entre as variáveis de composição corporal e o número de passos realizados por dia nas mulheres mais jovens e com a capacidade cardiorrespiratória nas voluntárias em idade mais avançada. Além disso, pode-se observar que em mulheres na pós-menopausa mais jovens a quantidade de gordura corporal pode influenciar no número de passos diário.

\begin{abstract}
Model of the study: Cross-sectional study. Objective: To verify the relationship between body fat, number of steps and cardiorespiratory fitness in postmenopausal women. Methods: 57 women over 50 years were divided into four groups according to the median of age $(<60.8$ and $\geq 60.8$ years) and median of body fat $(<22.8 \geq 33.2 \mathrm{Kg})$. The dual-energy X-ray absorptiometry (DXA) was used to estimate total fat mass, percentage of body fat and trunk fat. The test of six-minute walk was used to analyze the cardiorespiratory fitness and a tri-axial accelerometer to determinate the number of steps per day. Results: Younger women accumulated more steps per day $(<60.8=7727.5 x \geq 60.8=6173.2, p<0.014)$ and among the younger women group, the one with lower body fat accumulated more steps/day (lower fat $=8405$ steps $\mathrm{x}$ higher fat $=6118$ steps, $p<0.031)$. There was no significant difference between women with higher age and higher body fat. Regarding to the cardiorespiratory fitness, there was no significant difference between the groups. All variables of body fat presented low and negative relationship with both number of steps in the younger group and cardiorespiratory fitness in older age group. Conclusion: Body fat present low and negative correlation with the number of steps and cardiorespiratory fitness in postmenopausal women, furthermore, the age seems to influence this relationship.
\end{abstract}

Key words: Obesity. Accelerometry. Physical Activity. Postmenopause.

\section{Referênclas}

1. Donato GB, Fuchs SC, Oppermann K, Bastos C, Spritzer PM. Association between menopause status and central adiposity measured at different cutoffs of waist circumference and waistto-hip ratio. Menopause. 2006; 13:280-5.

2. Mattos $M$, Farinatti, P. Influência do treinamento aeróbio com intensidade e volume reduzidos na autonomia e aptidão físico-funcional de mulheres idosas. Rev Port Ciênc Desporto. 2007; 7 : 100-8.

3. Whitcroft SEA, Herriot. Insulin resistance and management of the menopause: a clinical hypothesis in practice. Menopause. 2011;17:24-8.

4. Gomes F, Telo DF, Souza HP, Nicolau JC, Halpern A, Serrano $\mathrm{CV}$. Obesity and coronary artery disease: role of vascular inflammation. Arq Bras Cardiol. 2010;94:255-61.

5. Mosca L, Appel LJ, Benjamin EJ, Berra K, Chandra-Strobos N, Fabunmi RP, et al. American Heart Association. Evidence- based guidelines for cardiovascular disease prevention in women. Circulation. 2004;109:672-93.

6. Vincent HK, Vincent KR, Lamb KM. Obesity and mobility disability in the older adult. Obes Rev. 2010;11:568-79.

7. Gabriel KP, Conroy MB, Schmid KK, Storti KL, High RR, Underwood DA, et al. The Impact of Weight and Fat Mass Loss and Increased Physical Activity on Physical Function in Overweight, Postmenopausal Women: Results from the WOMAN Study. Menopause. 2011;18:759-65.

8. Sutton-Tyrrell K, Zhao X, Santoro N, Lasley B, Sowers M, Johnston J, Mackey R, Matthews K. Reproductive hormones and obesity: 9 years of observation from the Study of Women's Health Across the Nation. Am J Epidemiol. 2010;171:120313.

9. Friedenreich CM, Woolcott CG, McTiernan A, Terry T, Brant R, Ballard-Barbash R, et al.. Adiposity changes after a 1-year aerobic exercise intervention among postmenopausal women: a randomized controlled trial. Int J Obes. 2011; 35:427-35. 
10. Organización Mundial De La Salud. Investigaciones sobre la menopausia en los años noventa. Genebra: Organización Mundial de la Salud;1996.

11. Freitas Junior IF. Padronização de técnicas antropométricas. São Paulo: Cultura Acadêmica: Universidade Estadual Paulista, Pró reitoria de graduação, 2009.

12. ATS Statement: guidelines for the six-minute walk test. Am J Respir Crit Care Med. 2002;166:111-7.

13. Nakamura F, Moreira A, Aoki M. Monitoramento da carga de treinamento: a percepção subjetiva do esforço da sessão é um método confiável? Rev educ Fís/UEM. 2010;21:1-11.

14. Douchi T, Yamamoto S, Yoshimitsu N, Andoh T, Matsuo T, Nagata Y. Relative contribution of aging and menopause to changes in lean and fat mass in segmental regions. Maturitas. 2002;30;42:301-6.

15. Buonani C, Rosa CS, Diniz TA, Christofaro DG, Monteiro HL, Rossi FE, et al. Physical activity and body composition in menopausal women. Rev Bras Ginecol Obstet. 2013;35:1538.

16. Ford ES, Li C, Zhao G, Pearson WS, Tsai J, Churilla JR. Sedentary behavior, physical activity, and concentrations of insulin among US adults. Metabolism. 2010;59(9):1268-75.
17. Woo J, Leung J, Kwok T. BMI, body composition, and physical functioning in older adults. Obesity (Silver Spring). 2007;15:1886-94.

18. Angleman SB, Harris TB, Melzer D. The role of waist circumference in predicting disability in periretirement age adults. Int J Obes. 2006;30:364-73.

19. Powers SK, Howley ET. Auxílios Ergogênicos. In: Powers SK, Howley ET. Fisiologia do Exercício - Teoria e Aplicação ao Condicionamento e ao Desempenho. 3 Ed. São Paulo: Manole, 2000.

20. Thomson JL, Landry AS, Zoellner JM, Tudor-Locke C, Webster M, Connell C, et al. Several steps/day indicators predict changes in anthropometric outcomes: HUB City Steps. BMC Public Health. 2012;12:983.

21. Graff SK, Alves BC, Toscani MK, Spritzer PM. Benefits of pedometer-measured habitual physical activity in healthy women. Appl Physiol Nutr Metab. 2012 Feb;37:149-56.

22. Shephard RJ. Limits to the measurement of habitual physical activity by questionnaires. Br J Sports Med. 2003;37:197-206.

23. Woodrow G. Body composition analysis techniques in the aged adult: indications and limitations. Curr Opin Clin Nutr Metab Care. 2009;12:8-14 